\title{
Targeting oral cancer stemness and chemoresistance by isoliquiritigenin-mediated GRP78 regulation
}

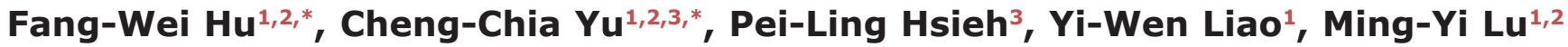 \\ and Pei-Ming $\mathrm{Chu}^{4}$ \\ ${ }^{1}$ School of Dentistry, Chung Shan Medical University, Taichung, Taiwan \\ ${ }^{2}$ Department of Dentistry, Chung Shan Medical University Hospital, Taichung, Taiwan \\ ${ }^{3}$ Institute of Oral Sciences, Chung Shan Medical University, Taichung, Taiwan \\ ${ }^{4}$ Department of Anatomy and Graduate Institute of Biomedical Sciences, School of Medicine, China Medical University, \\ Taichung, Taiwan \\ *These authors have contributed equally to this work \\ Correspondence to: Pei-Ming Chu, email: pmchu@mail.cmu.edu.tw \\ Keywords: oral squamous cell carcinomas, isoliquiritigenin, cancer stemness, GRP78, chemoresistance \\ Received: August 01,2017 Accepted: August 28, $2017 \quad$ Published: September 28, 2017 \\ Copyright: $\mathrm{Hu}$ et al. This is an open-access article distributed under the terms of the Creative Commons Attribution License 3.0 \\ (CC BY 3.0), which permits unrestricted use, distribution, and reproduction in any medium, provided the original author and source \\ are credited.
}

\section{ABSTRACT}

Cancer stem cells (CSCs) are cells that drive tumorigenesis, contributing to metastasis and cancer recurrence as well as resistance to chemotherapy of oral squamous cell carcinomas (OSCC). Therefore, approaches to target CSCs become the subject of intense research for cancer therapy. In this study, we demonstrated that isoliquiritigenin, a chalcone-type flavonoid isolated from licorice root, exhibited more toxicity in oral cancer stem cells (OSCC-CSCs) compared to normal cells. Treatment of isoliquiritigenin not only inhibited the self-renewal ability but also reduced the expression of CSC markers, including the ALDH1 and CD44. In addition, the capacities of OSCC-CSCs to invade, metastasize and grow into a colony were suppressed by isoliquiritigenin. Most importantly, we showed that isoliquiritigenin potentiated chemotherapy along with downregulated expression of an ABC transporter that is associated with drug resistance, ABCG2. Moreover, a combination of isoliquiritigenin and Cisplatin significantly repressed the invasion and colony formation abilities of OSCC-CSCs. Our results suggested that administration of isoliquiritigenin reduced the protein expression of mRNA and membrane GRP78, a critical mediator of tumor biology. Overexpression of GRP78 reversed the inhibitory effect of isoliquiritigenin on OSCC-CSCs. Furthermore, isoliquiritigenin retarded the tumor growth in nude mice bearing OSCC xenografts. Taken together, these findings showed that isoliquiritigenin is an effective natural compound that can serve as an adjunct to chemotherapy for oscC.

\section{INTRODUCTION}

Head and neck squamous cell carcinoma (HNSCC) is one of the most prevalent and aggressive malignancies worldwide with a dismal prognosis. The majority of HNSCC is oral squamous cell carcinoma (OSCC) that arises from the mucosal surfaces of the oral cavity, lateral border of the tongue, and floor of the mouth. Over the past few years, the survival rate is still far from satisfactory despite continuous efforts to develop effective treatments [1]. Moreover, recurrence of cancer after chemotherapy remains a major problem that needs to be solved [2]. A recent study has revealed that 3-year overall survival rate of advanced OSCC patients with recurrent tumors was less 
than $30 \%$ [3]. Hence, it is imperative to identify targets and develop novel therapeutic strategies for OSCC.

Resistance to radio/ chemotherapy has been attributed to cancer stem cells (CSCs) [4], which possess tumor-initiating, self-renewal properties and give rise to nontumorigenic progeny [5]. It has been suggested that CSCs play a pivotal role in the relapse, metastasis and therapeutic refractoriness of tumors [6]. Various studies have shown that implementation of treatments against CSCs suppress chemoresistance [7, 8] and increase the effectiveness of conventional therapy [9] across a spectrum of malignancies. Thus, CSC-focused therapy seems destined to be the core of effective anticancer approaches.

Isoliquiritigenin (ISL), a flavonoid from licorice, has been found to exhibit numerous pharmaceutical capacities, such as spasmogenic [10], spasmolytic [1014], analgesic [12] and anti-inflammatory [15] properties. In addition, accumulating evidence has demonstrated the significant anti-tumor ability of ISL. It has been shown to inhibit angiogenesis via VEGF/VEGFR-2 pathway [16] and induce apoptosis through suppression of COX2 [17], increase in cyclin-dependent kinase 2 activity [18], or generation of reactive oxygen species [19] in cancer cells. Moreover, it has been found to suppress endometrial [20] or lung [21] tumor growth in vivo. In HNSCC, ISL has been demonstrated to inhibit tongue squamous carcinoma cells via the anti-oxidant mechanism [22]. Our previous work also has shown that ISL induces cell cycle arrest, apoptosis, and DNA damage in OSCC cells [23]. Moreover, the DNA repair-associated ataxia telangiectasia mutated (ATM) and phospho-ATM were found downregulated. We demonstrated that low dose of ISL suppressed the malignant phenotypes of OSCCs in vitro and retarded tumor growth in vivo. Above all, it has been reported that ISL inhibits breast CSCs [24] and chemosensitizes these CSCs via targeting glucoseregulated protein (GRP-78) [25]. Nevertheless, the antiCSC effect or chemosensitizing potential of ISL on OSCC remains to be determined.

GRP78/BiP/HSPA5 is a major chaperone in the endoplasmic reticulum (ER), which regulates a number of biological functions including tumor proliferation [26] and metastasis [27] as well as chemo/radioresistance [28]. Although GRP78 is located mainly in the ER, it has been reported to be anchored at the cell membrane [29]. Cell surface GRP78 has been found to promote cell proliferation by ERK1/2, p38 MAPK and PI-3K and cell survival by Akt and NF-kB signaling cascades in prostate cancer cells [30]. It has been shown that ISL exerts chemosensitizing effects via GRP78 as GRP78 is the direct target of ISL in inhibiting $\beta$-catenin/ABCG2 signaling [25]. In addition, $\beta$-catenin signaling has been found to be the key to maintain self-renewal and tumorigenicity of CSCs in HNSCC [31]. Accordingly, it is crucial to examine whether ISL could inhibit OSCC-
CSCs or potentiate chemotherapy via downregulation of GRP78. To this end, we assessed the influence of ISL on stemness features of OSCC-SCSs and then evaluated its effect on improvement of chemotherapy. Subsequently, we examined the expression of GRP78 in OSCC-CSCs and tested whether the stemness characteristics were affected following overexpression of GRP78. The tumor transplantation mouse model was utilized as well to confirm the role of GRP78 in vivo.

\section{RESULTS}

\section{Cytotoxic effects of isoliquiritigenin in oral cells}

We first investigated the impact of ISL on cell viability of an immortalized normal oral epithelial cells (SG) and CSCs-derived from these oral cancer cell lines (SAS and OECM-1) using MTT assay. As shown in Figure 1A, the cytotoxic effect of ISL was more potent in two CSCs than normal SG cells. After treatment with ISL for $24 \mathrm{hr}$, the half maximal effective dose (IC50) of ISL deduced from the generated dose-response curve to SG cells, SAS-CSCs, OECM-1-CSCs were $386.3 \pm 29.7$ $\mu \mathrm{M}, 144.9 \pm 25.7 \mu \mathrm{M}$ and $104.5 \pm 26.2 \mu \mathrm{M}$, respectively. This finding revealed that the more cytotoxic ISL was to OSCC-CSCs as it required a higher concentration to suppress normal SG cells.

\section{Self-renewal capacity of CSCs is hindered by addition of isoliquiritigenin}

The ability to self-renew is an important feature of CSCs [32] and secondary sphere formation assay has been used widely to evaluate this property $[33,34]$. Our results showed that maintenance of self-renewal in these two OSCC-CSCs was inversely affected as the concentration of isoliquiritigenin increased (Figure 1B), suggesting the potential anti-OSCC-CSCs effect of ISL. Consequently, we assessed other stemness signatures in the following experiments.

\section{Isoliquiritigenin effectively eliminates ALDH1 enzymatic activity and CD44 positivity in OSCC- CSCs}

Aldehyde dehydrogenase 1 (ALDH1) is a cytosolic isoenzyme that is responsible for the oxidation of retinol to retinoic acid during early stem cell differentiation and has been proven to be a CSC marker in HNSCC $[35,36]$. A commonly used method to identify CSCs is via the Aldefluor Assay (Stemcell Technologies, Inc.) that measures ALDH1 activity with $\mathrm{N}, \mathrm{N}$-diethylaminobenzaldehyde (DEAB) as a negative control compound [37]. We observed that ISL induced a concentration-dependent decrease in activity of ALDH1 of both OSCC-CSCs (Figure 2A). Additionally, we 
A
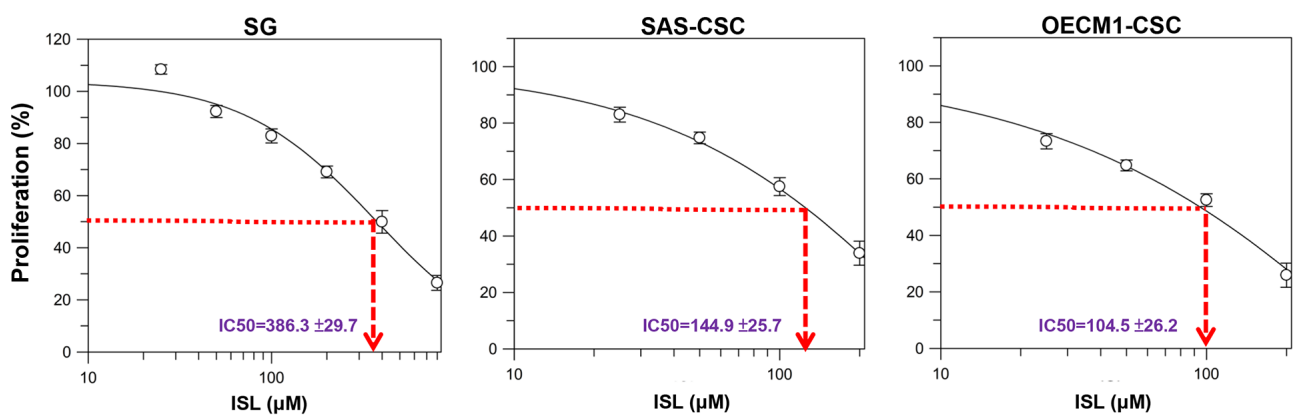

B
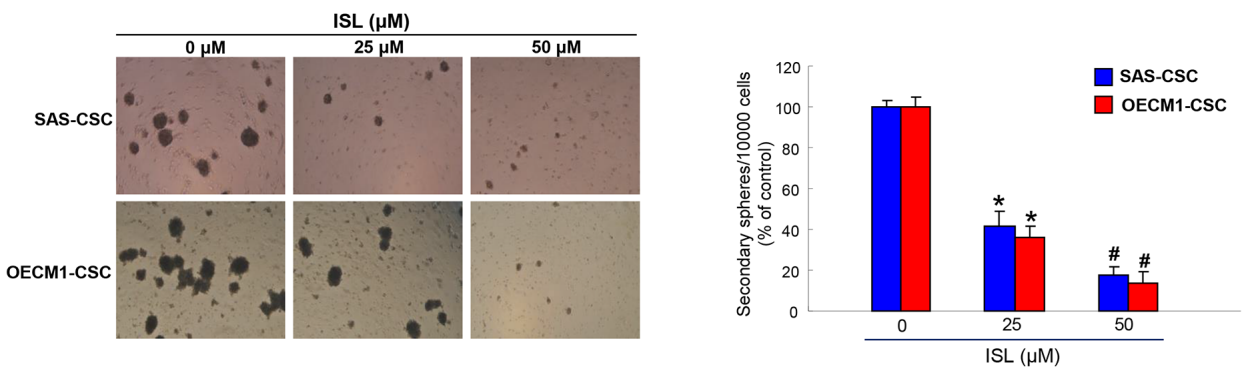

Figure 1: Effects of ISL on cell viability and self-renewal of OSCC-CSCs. (A) Normal SG cells and OSCC-CSCs derived from SAS and OECM1 cell lines were treated with various concentration of ISL. Cell viability was determined using MTT assay and IC50 values for SG, SASCSCs and OECM1-CSCs were 386.3 $\pm 29.7,144.9 \pm 25.7$ and 104.5 $\pm 26.2 \mu \mathrm{M}$, respectively. (B) Secondary sphere formation was utilized to assess the self-renewal capacity of OSCC-CSCs with indicated concentration of ISL. The number of the secondary sphere was counted and presented as the percentage control. ${ }^{*} p<0.05$ compared to no treatment control group. ${ }^{*} p<0.05$ compared to $25 \mu \mathrm{M}$ treatment group or no treatment control group.

A
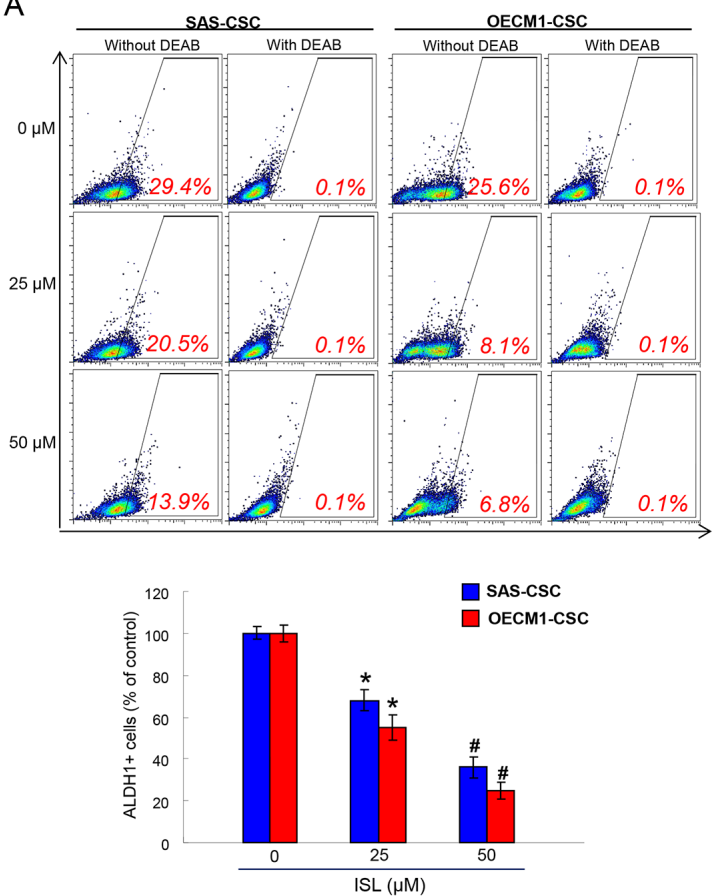

B
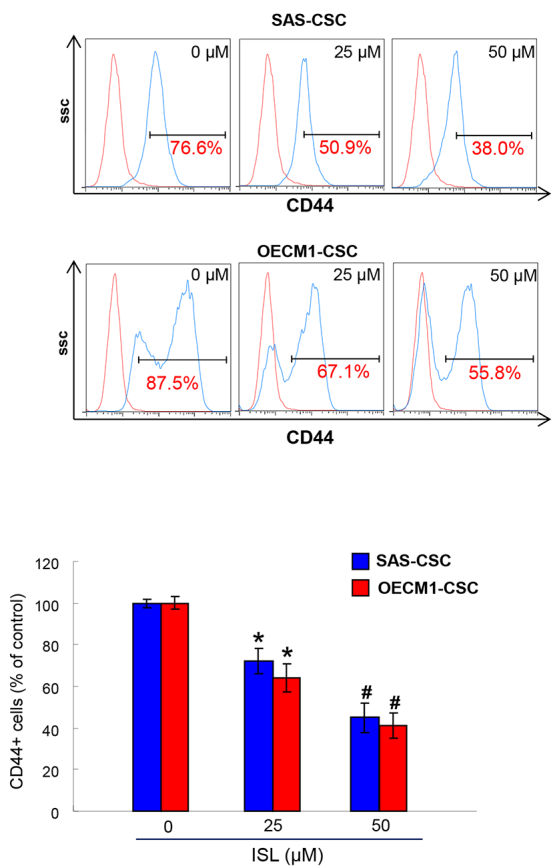

Figure 2: The ALDH1 enzymatic activity and CD44 positivity were reduced with ISL treatment. ALDH1 enzymatic activity (A) and CD44 positivity (B) was gradually eliminated in OSCC-CSCs as the concentration of ISL increased by flow cytometry analysis. Results are means \pm SD. ${ }^{*} p<0.05$ compared to no treatment control group. ${ }^{*} p<0.05$ compared to $25 \mu \mathrm{M}$ treatment group or no treatment control group. 
examined the expression of CD44, which is also a highly selective marker for CSCs in HNSCC [38]. Flow cytometry analysis of CD44 positivity indicated that ISL-treated OSCC-CSCs expressed a dose-dependently reduction in CD44 expression (Figure 2B). Together, we showed that CSCs markers were gradually downregulated in both OSCC-CSCs along with the increase in isoliquiritigenin concentration.

\section{Isoliquiritigenin attenuates the oncogenicity abilities in OCSCs}

Given that CSCs exhibit high tumor-initiating and metastasis capacities and are associated with therapeutic refractoriness of cancers $[6,39]$, we sought to measure the effects of ISL on colony forming, migration and invasion abilities of OSCC-CSCs. Single-cell suspensions of ISL-treated OSCC-CSCs were used for analysis of their

A
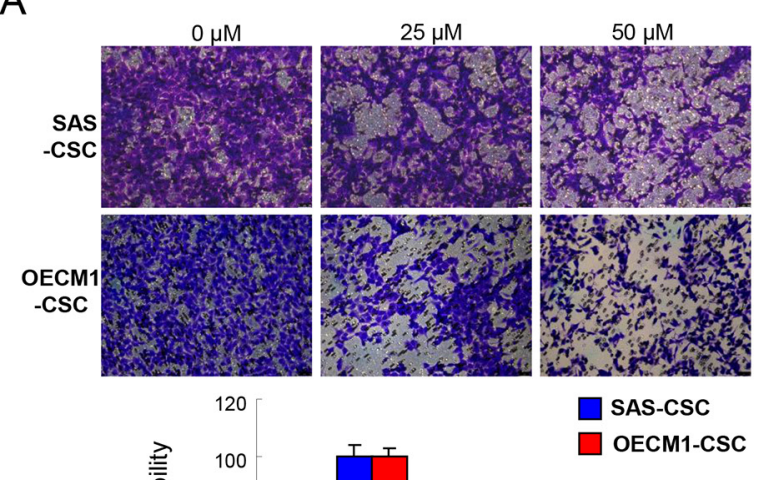

C

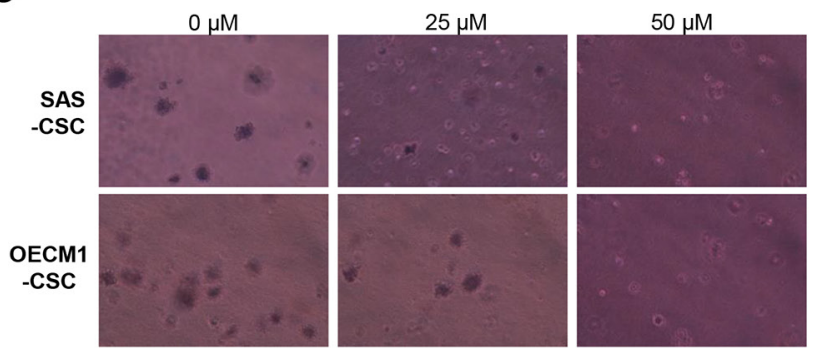

metastatic capacity in vitro as described in the Methods section. Our data suggested that ISL dose-dependently suppressed the migration (Figure 3A) and invasion (Figure 3B) abilities of OSCC-CSCs. Anchorage-independent growth assay showed that colony formation capacity of OSCC-CSCs was inhibited by ISL (Figure 3C). These results indicated that ISL significantly suppressed the cologenicity and metastasis potential of OSCC-CSCs.

\section{Isoliquiritigenin potentiates chemotherapy in the treatment of OSCC}

Drug resistance is a crucial cause of treatment failure [40] and it has been attributed to the existence of CSCs [41]. As expected, the cell survival of OSCC-CSCs was barely affected by Cisplatin compared with nonCSCs, while the combination of ISL markedly repressed the number of CSCs (Figure 4A). ABCG2, an ATP-

B
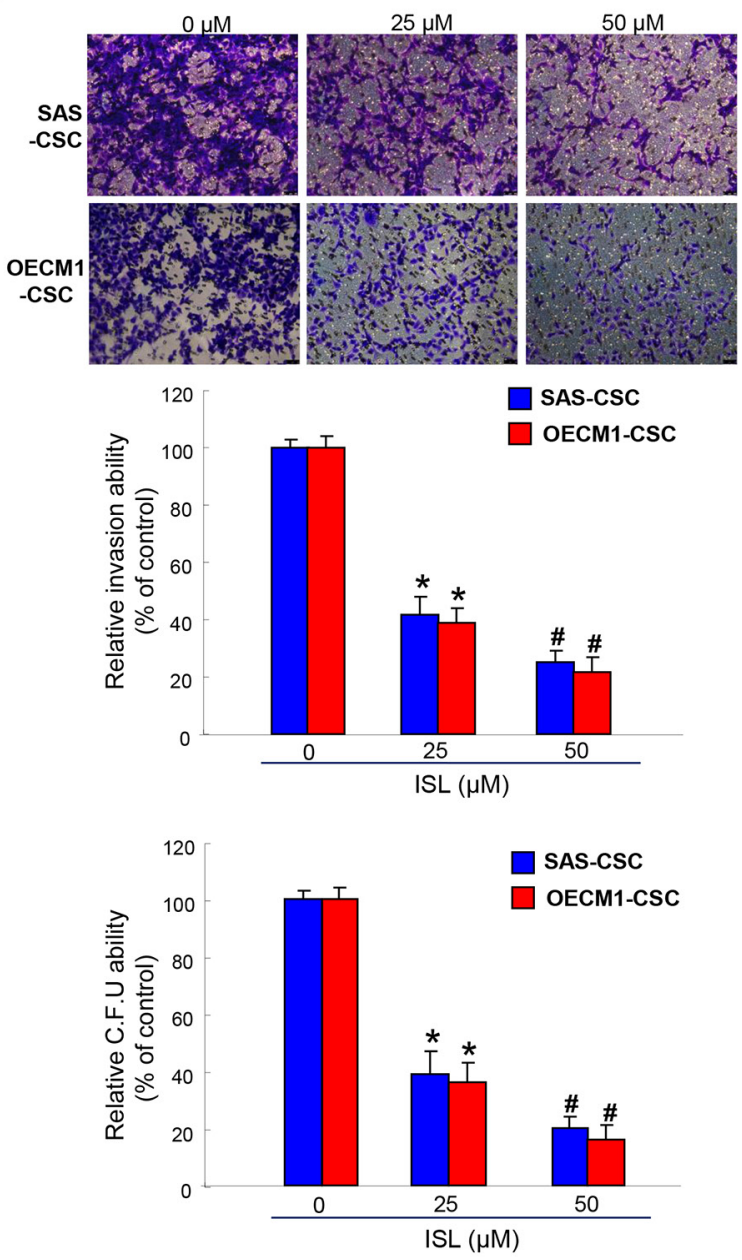

Figure 3: The oncogenicity of OSCC-CSCs was suppressed by ISL. Representative images (upper) and quantification (lower) of (A) migration assay and (B) Matrigel invasion assay of OCSCs treated with various concentration of ISL. (C) OCSC with dose-dependent ISL treatment were assigned for the colony formation assay. Experiments were performed in triplicate. Values are expressed as mean \pm SD. ${ }^{*} p<0.05$ compared to no treatment control group. ${ }^{*} p<0.05$ compared to $25 \mu \mathrm{M}$ treatment group or no treatment control group. 
binding cassette transporter protein, is not only a stem cell marker but also associated with drug resistance since ABCG2 has the capacity to transport a broad range of substrates [40-43]. We showed that expression of ABCG2 was downregulated with the increased concentration of ISL (Figure 4B). Thereafter, we assessed the colony formation and invasion capacities to investigate whether ISL could enhance the effect of Cisplatin. Results of these two analyses showed that administration of ISL alone or combined with Cisplatin both reduced the colony forming and invasion abilities of OSCC-CSCs (Figure 4C and 4D). Collectively, we demonstrated that ISL possesses the potential to serve as an adjunct to chemotherapy.

\section{Anti-CSCs effect of isoliquiritigenin is via downregulation of GRP78}

Our previous work has revealed that the transcripts and protein levels of GRP78 were significantly upregulated

A

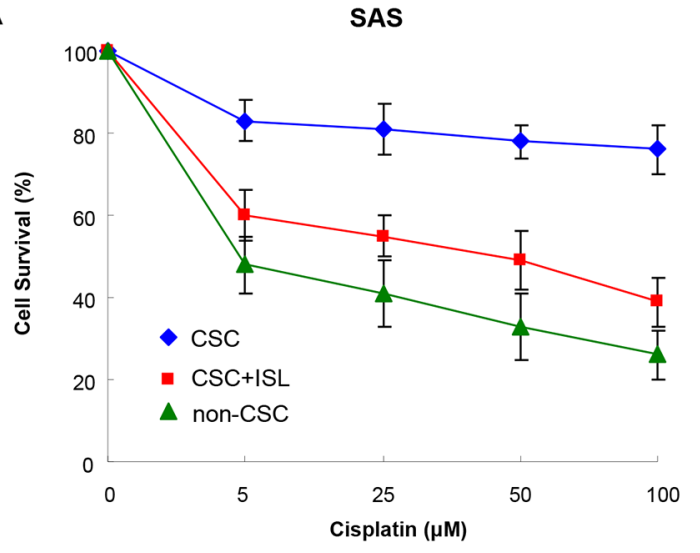

C

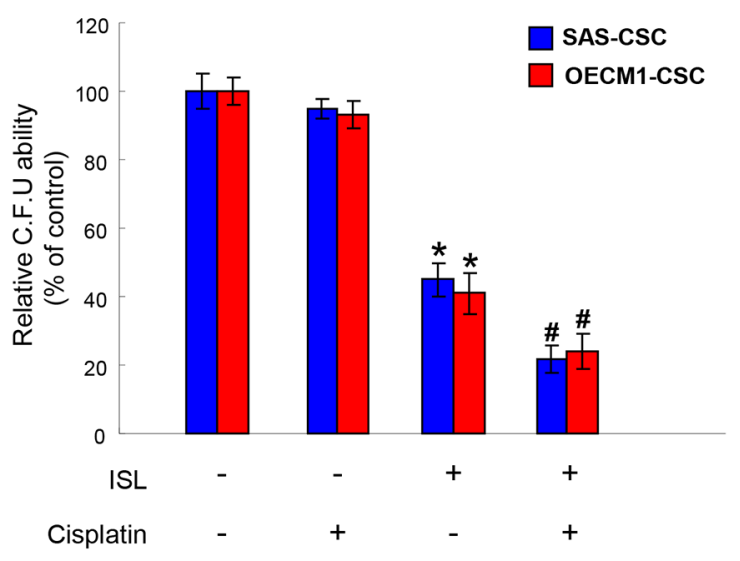

in enriched OSCC-CSCs [44]. To elucidate the molecular mechanisms by which to mediate the anti-CSCs effect of ISL, we evaluated the expression of GRP78 after treatment of ISL. Protein expression level of GRP78 was reduced following increased concentration of ISL (Figure 5A). Likewise, membrane-associated GRP78 positive $\left({ }^{\mathrm{mem}} \mathrm{GRP} 78^{+}\right)$cells in OSCC-CSCs were also downregulated in response to the addition of ISL by flow cytometry analysis (Figure 5B). Moreover, we found that the overexpression of GRP78 reversed the inhibitory effect of ISL on colony forming (Figure 5C) and invasion (Figure 5D) abilities of OSCC-CSCs. These results suggested that the ISL-inhibited CSCs effect was associated with GRP78.

\section{Administration of isoliquiritigenin suppressed the tumor growth in vivo}

Tumorigenesis in vivo is a significant aspect when evaluating the anti-CSC effect. Our results showed that

B

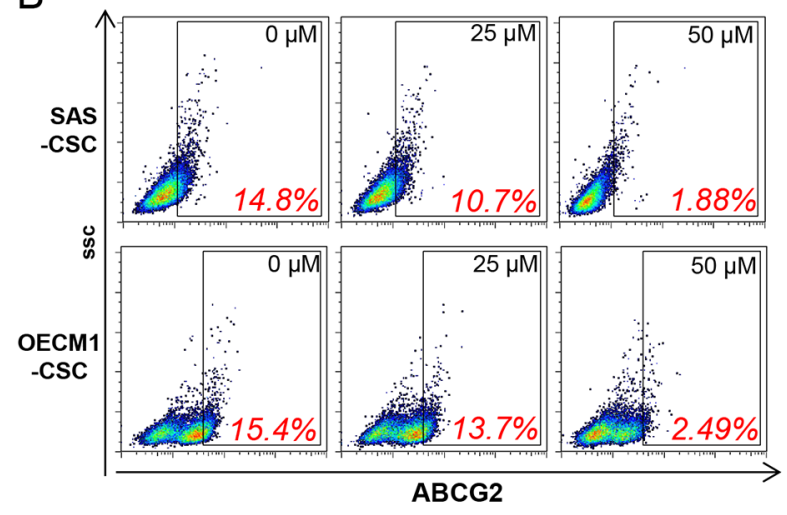

$\mathrm{D}$

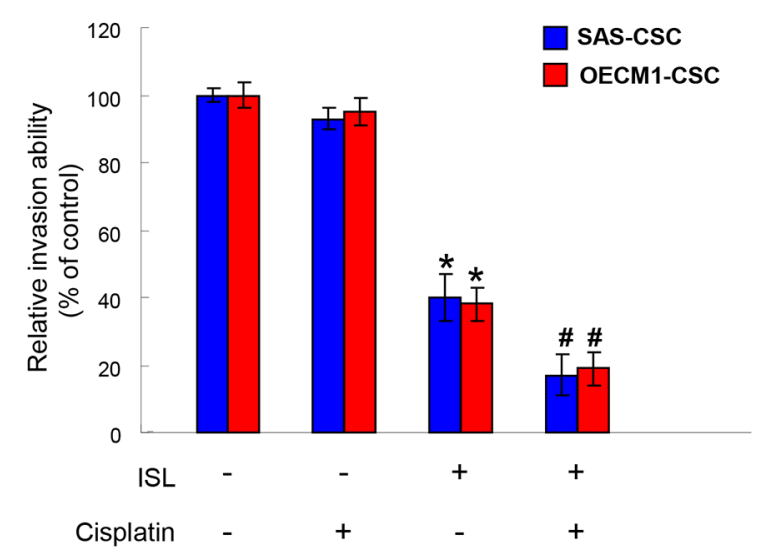

Figure 4: Chemoresistance in OSCC-CSCs is attenuated with ISL treatment. (A) Cell survival of non-CSCs, CSCs and CSCs+ ISL after Cisplatin were examined using MTT assay. (B) The expression of ABCG2 in two OSCC-CSCs was tested by flow cytometry following treated with various concentration of ISL. (C) Colony formation and (D) invasion capacities after Cisplatin treatment were evaluated with or without ISL. Data were shown as the mean \pm SD. ${ }^{*} p<0.05$ compared to no treatment control group. ${ }^{\#} p<0.05$ compared to Cisplatin alone group. 
oral administration of ISL successfully delayed the tumor growth after transplanting tumor cells into nude mice (Figure 6A). Furthermore, we demonstrated that the expression of GRP 78 was decreased in the tumor tissues extracted from the ISL-treated group by immunoblotting (Figure 6B), suggesting the tumor-suppressive potential of ISL in vivo.

\section{DISCUSSION}

Accumulating evidence has attributed the chemotherapy failure or metastasis to the existence of CSCs $[45,46]$. In this study, we examined the effect of ISL on the self-renewal, metastatic capacities, and sensitization of chemotherapy of OSCC-CSCs. We found that ISL reduced the expression of CSC markers, the ability of selfrenewal and colony formation by reducing the expression of GRP78. Furthermore, our findings suggested ISL enhanced the treatment effect of Cisplatin with reduced CSCs properties and ABCG2 expression. Numerous studies have shown that $\mathrm{ABCG} 2$ expression is associated

A
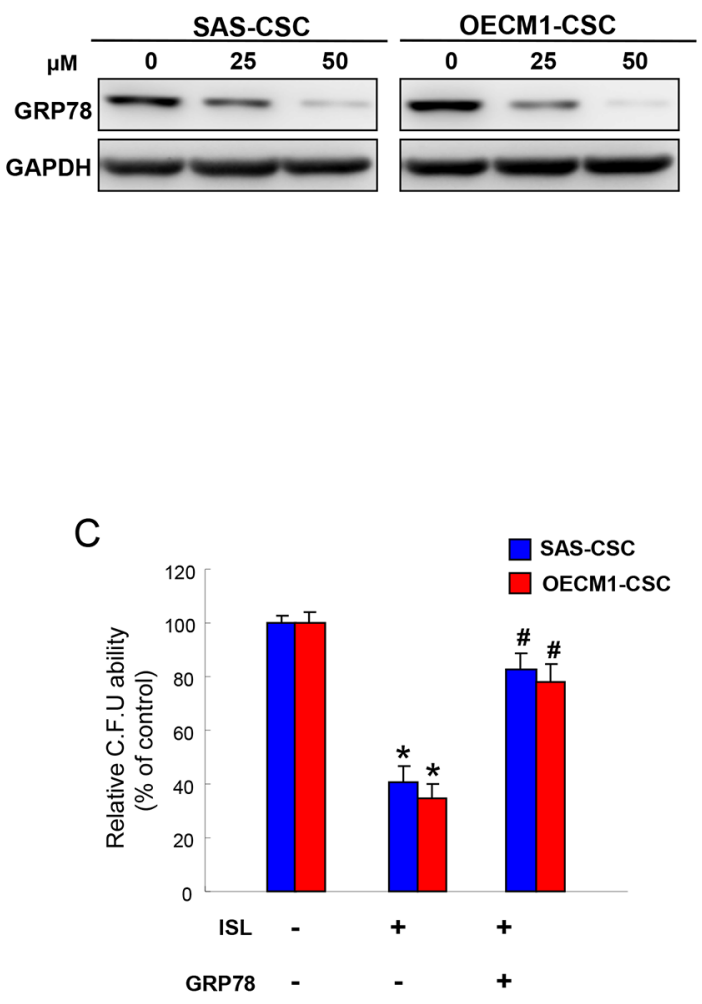

with stemness and drug resistance [40-43], which may lead to treatment failure. The reduced ABCG2 expression following ISL treatment in our study was in accordance with a previous report showing ISL chemosensitizes breast CSCs via GRP78/ $\beta$-catenin/ABCG2 signaling.

Several studies have suggested that GRP78 was crucial for enhancement of tumor cell proliferation, protection against apoptosis, and promotion of tumor angiogenesis [47-50]. Our previous study has identified GRP78 as a putative candidate on mediating the stemness hallmarks of OSCC-CSCs by differential systemic analyses. We showed that ${ }^{\mathrm{mem}} \mathrm{GRP} 78^{+}$OSCCCSCs displayed stemness properties of self-renewal and radioresistance. In addition, we demonstrated that downregulation of GRP78 inhibited tumorigenicity, whereas overexpression of GRP78 in OSCC-CSCs enhanced the malignant potentials and ${ }^{\mathrm{mem}} \mathrm{GRP} 78^{+}$ expression profile [44]. In the current study, we utilized ISL-mediated downregulation of GRP78 to suppress the oncogenicity of OSCC-CSCs in vitro and in vivo. In consistent with other studies showing silencing of
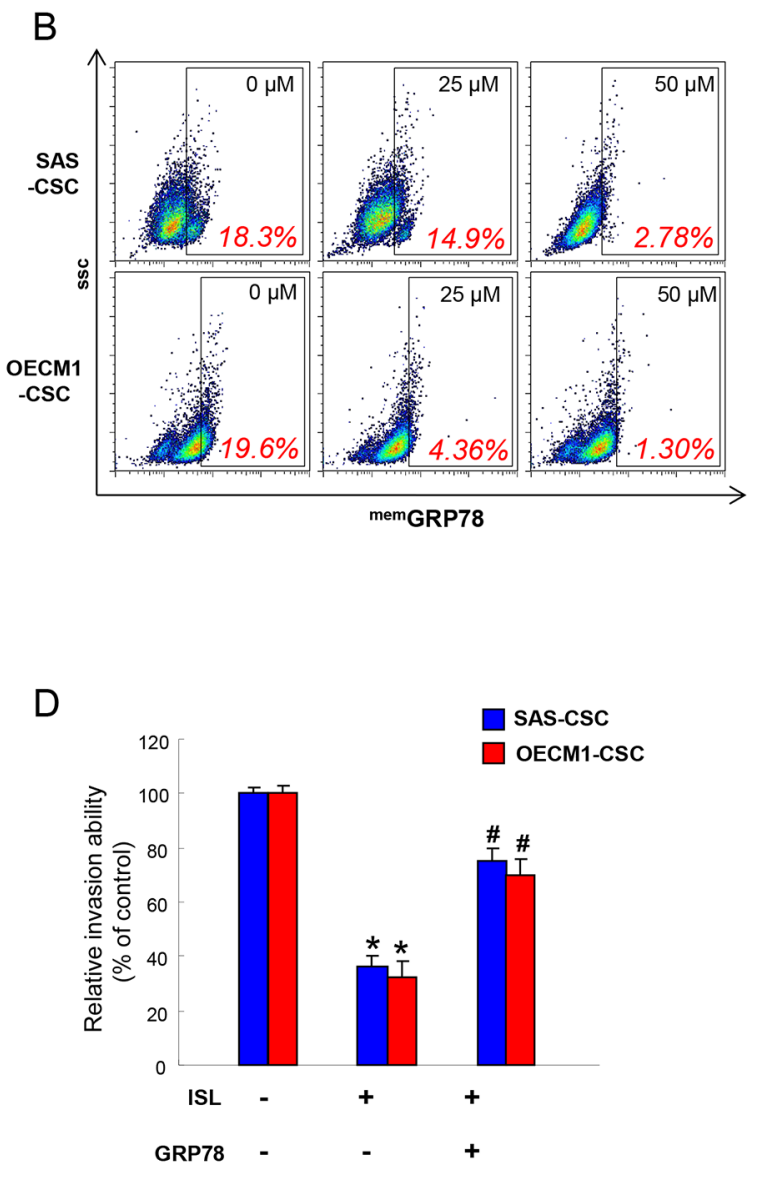

Figure 5: The anti-tumor effect of ISL is mediated by GRP78. (A) Protein levels of GRP78 in OCSCs were evaluated in response to ISL treatment by western blotting. (B) Membrane-associated GRP78 positive $\left({ }^{\mathrm{mem}} \mathrm{GRP} 78^{+}\right)$cells in OSCC-CSCs were analyzed in OCSCs with ISL treatment by flow cytometry analysis. Clonogenicity $(\mathbf{C})$ and invasion (D) capabilities of OCSCs were examined in the absence or presence of ISL and GRP78. Data were shown as a percentage of no treatment group. ${ }^{*} p<.05$ compared to no treatment group; ${ }^{\#} \mathrm{p}<.05$ compared to ISL only group. 
GRP78 decreases cell growth and sensitizes cancer cells to chemotherapy $[51,52]$, our results proved that downregulation of GRP78 by administration of ISL also enhanced the treatment effect of Cisplatin (Figure 4). A limitation of the current study was the xenotransplantation model of OSCCs, further studies may utilize carcinogenic agents to induce oral cancer in animals and evaluate the effect of ISL on prevention of OSCCs development.

It has been shown that ISL induced growth inhibition and apoptosis in human breast cancer cells via deactivation of PI3-K/Akt pathway [53] or in endometrial cancer via activation of ERK [20]. In this study, we found that ISL reduced the expression of GRP78, and decreased expression of GRP78 has been found to exert pro-proliferative and anti-apoptotic properties in prostate carcinoma cells through activation of MAPK and PI3-K pathways [30]. Another study has revealed that Cripto/ GRP78 complex mediated oncogenic signaling via
MAPK, PI3-K and Smad2/ 3 pathways in mammary cells [54]. Likewise, blockage of GRP78 expression suppresses tumorigenesis and Akt activation with PTEN loss in prostate epithelium [55]. As for OSCC-CSCs, we have shown that knockdown of GRP78 induced apoptosis via enhanced expression of PTEN, BAX, and caspase 3 as well as reduced expression of $\mathrm{p}-\mathrm{MAPK}$ in our previous work [44]. Further studies are required to clarify whether PI3-K/ Akt or MAPK pathways are involved in the anti-CSCs effect following ISL-mediated GRP78 downregulation.

In conclusion, we demonstrated that ISL has the potential to serve as an anti-CSCs agent with its inhibitory effect on the CSC characteristics, including the expression of CSC markers, self-renewal, metastasis, colony formation capacities as well as enhancement of chemotherapy. Our results suggested that ISL-mediated reduction of GRP78 in OSCC-CSCs played a critical role in these phenomena. Furthermore, we showed that oral
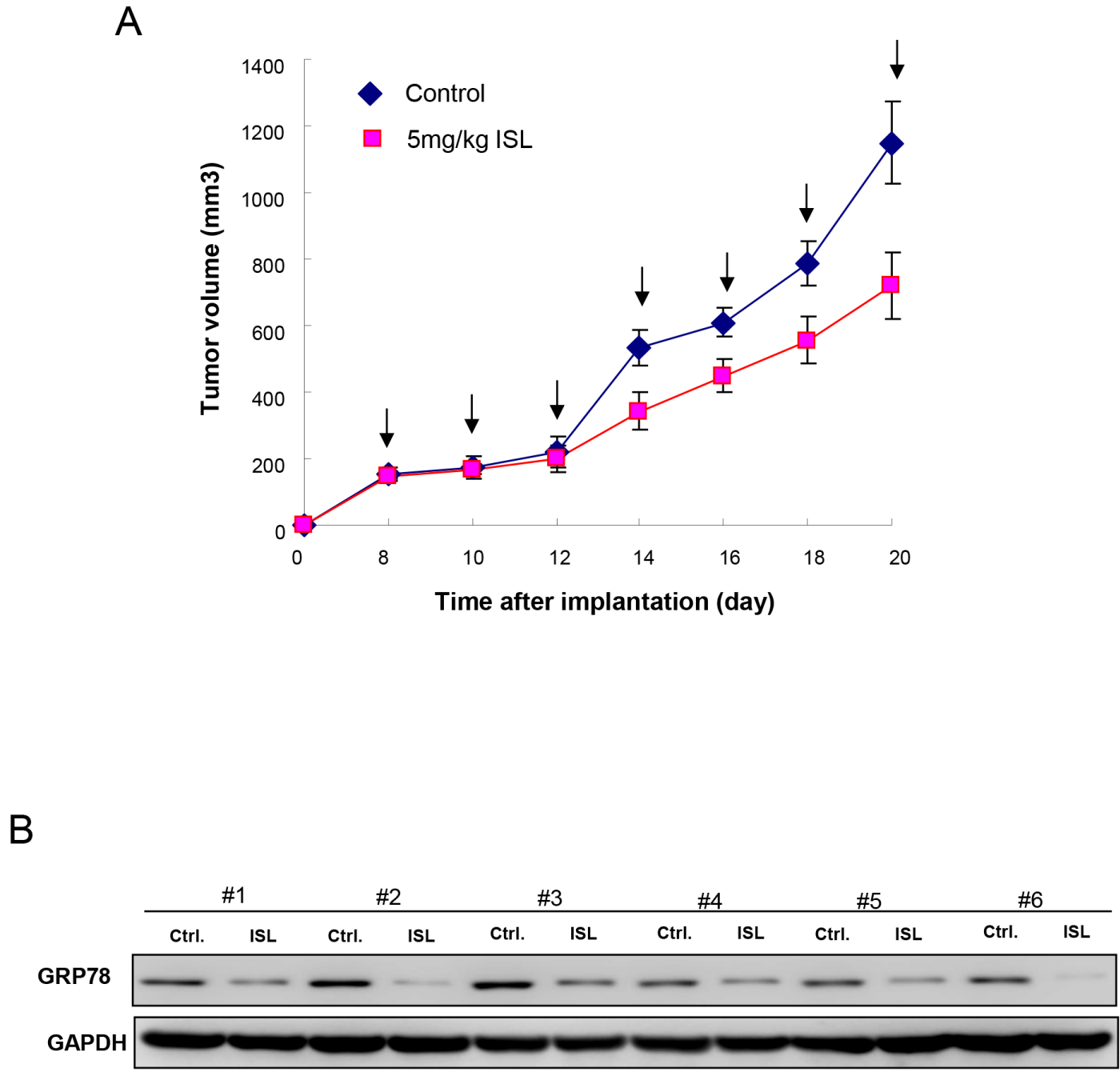

Figure 6: Therapeutic efficiency of ISL in OCSC-transplanted mice. (A) Tumor size was evaluated in control and ISL treatment $(5 \mathrm{mg} / \mathrm{kg})$ groups over time until day 20. (B) After sacrifice, the expression of GRP78 in the excised tumors from both groups ( $\mathrm{n}=6$ for each group) was detected using Western blot. Values are expressed as mean \pm SD. ${ }^{*} p<.05$ compared to control. 
administration of ISL successfully retarded tumor growth in xenotransplanted mice. Altogether, these findings support that ISL may be used as a nutritional intervention for an adjunct to chemotherapy in clinical application.

\section{MATERIALS AND METHODS}

\section{Reagents and cell lines cultivation}

ISL was purchased from Sigma-Aldrich (St. Louis, MO, USA). SG cells (Smulow-Glickman human gingival epithelioid cells) were cultured in Dulbecco modified Eagle's medium (DMEM) containing 10\% fetal bovine serum (FBS). SAS cells (high-grade human tongue carcinoma cell line) were cultured in DMEM supplement with 10\% FBS and F-12. OECM-1 cells (oral gingival squamous cell carcinoma cell line) were grown in RPMI supplemented with 10\% FBS using previously described protocols [56]. All cells were maintained in an incubator with $5 \% \mathrm{CO}_{2}$ and $95 \%$ humidity at $37^{\circ} \mathrm{C}$.

\section{Cell viability}

SG, SAS, or OECM-1 cells were plated in 96well plates at $1 \times 10^{4}$ cells/well in $0.1 \%$ DMSO or various concentration of ISL-containing medium (25, $50,100,200$ to $400 \mu \mathrm{M}$ ) and cultured at $37^{\circ} \mathrm{C}$ for $24 \mathrm{~h}$. Cell proliferation/ survival was determined by MTT (3-(4,5-dimethylthiazol-2-yl)-2,5-diphenyl tetrazolium bromide) assay. The 570-nm absorbance of the DMSOtreated group was set as $100 \%$ and data were presented as a percentage of DMSO control [57].

\section{Secondary sphere formation}

OSCC cells were dissociated and cultured as tumorspheres in modified DMEM/F-12 supplemented with $\mathrm{N} 2$ (R\&D), $10 \mathrm{ng} / \mathrm{mL}$ epidermal growth factor (EGF, Invitrogen, Carlsbad, CA, USA), $10 \mathrm{ng} / \mathrm{mL}$ basic fibroblast growth factor (bFGF, Invitrogen), and penicillin/ streptomycin at $10^{3}$ live cells/low-attachment 6-well plate (Corning, Acton, MA, USA). The medium was changed every other day until the tumor sphere formation was observed in about 2 weeks [58].

\section{Aldefluor assay and flow cytometry analysis}

To measure the ALDH1 activity, Aldefluor assay was performed according to manufacturer's (Stemcell Technologies, Durham, NC, USA) instruction. Dissociated single cells were suspended in Aldefluor assay buffer containing ALDH substrate, and ALDH inhibitor, diethylaminobenzaldehyde (DEAB), was used as negative control.

Cells were stained with anti-CD44 (Miltenyi Biotech., Auburn, CA, USA), or anti- ${ }^{\text {mem GRP78 }}$
(Cell Signaling, Danvers, MA, USA) or anti-ABCG2 (Santa Cruz Biotechnology, Santa Cruz, CA, USA) antibodies conjugated to phycoerythrin according to the manufacturer's instructions. Red $(>650 \mathrm{~nm})$ fluorescence emission from 10,000 cells illuminated with blue $(488 \mathrm{~nm})$ excitation light was measured with FACSCalibur (Becton Dickinson, Mountain View, CA, USA) using CellQuest software [44, 57].

\section{Migration/ invasion assays}

$1 \times 10^{5}$ cells in medium with lower serum $(0.5 \%$ FBS) or various concentration of ISL were seeded into the top chamber of a transwell (Corning, Acton, MA, USA) with a porous transparent polyethylene terephthalate membrane $(8.0 \mu \mathrm{m}$ pore size $)$. Medium supplemented with higher serum $(10 \% \mathrm{FBS})$ was used as a chemoattractant in the lower chamber. For invasion assay, the membrane was coated with Matrigel ${ }^{\mathrm{TM}}$ (BD Pharmingen, NJ, USA). Cells were incubated for $24 \mathrm{~h}$ and cells that did not migrate through the pores were removed by a cotton swab. The migrated cancer cells were then visualized and counted from 5 randomly selected fields under 100-fold magnification using an inverted microscope [59].

\section{Anchorage-independent growth assay}

Each well of a 6-well plate was coated with $2 \mathrm{ml}$ bottom agar (Sigma-Aldrich) mixture (DMEM, 10\% (v/v) FCS, 0.6\% (w/v) agar). After the bottom layer was solidified, $2 \mathrm{ml}$ top agar-medium mixture (DMEM, 10\% $(\mathrm{v} / \mathrm{v}) \mathrm{FCS}, 0.3 \%(\mathrm{w} / \mathrm{v})$ agar) containing $2 \times 10^{4}$ cells and various concentration of ISL was added, and the plates were incubated at $37^{\circ} \mathrm{C}$ for 4 weeks. Plates were stained with $0.005 \%$ Crystal Violet and the number of total colonies with a diameter $\geq 100 \mu \mathrm{m}$ was counted over five fields per well for a total of 15 fields in triplicate experiments [60].

\section{RNA isolation and quantitative real-time reverse-transcriptase (RT)-PCR}

RNA was extracted from cells using Trizol reagent (Invitrogen) and reverse-transcribed using the Superscript III first-strand synthesis system (Invitrogen). Amplification was carried out on an ABI StepOne ${ }^{\text {TM }}$ Real-Time PCR Systems (Applied Biosystems, Carlsbad, CA, USA) using SYBR green I (Roche Molecular Systems, Alameda, CA, USA). PCR reactions were prepared in duplicate and heated to $95^{\circ} \mathrm{C}$ for $10 \mathrm{~min}$ followed by 40 cycles of denaturation at $95^{\circ} \mathrm{C}$ for $10 \mathrm{~s}$, annealing at $55^{\circ} \mathrm{C}$ for $5 \mathrm{~s}$, and extension at $72^{\circ} \mathrm{C}$ for $20 \mathrm{~s}$. Standard curves (cycle threshold values versus template concentration) were prepared for each target gene and for the endogenous reference (GAPDH) in each sample. All reagents and protocols were from Applied 
Biosystems, and detection was performed using 7900HT fast real-time PCR system [61].

\section{Stable overexpression of GRP78 in OSCC-CSCs}

The cDNA fragments encoding full-length GRP78 was cloned into the pLV-EF1 $\alpha$-MCS-IRES-GFP vector from Biosettia Inc. (Biosettia, San Diego, CA, USA). Lentivirus production was performed by co-transfection of plasmid DNA mixture with lentivector plus helper plasmids (VSVG and Gag-Pol) into 293T cells (American Type Culture Collection, Manassas, VA, USA) using Lipofectamine 2000 (LF2000, Invitrogen, Carlsbad, CA, USA) as previously described [62]. Stable GRP78overexpressing OCSCs were further purified by cell sorting with GFP positive cells.

\section{Bioluminescence imaging measurement of tumor growth in vivo}

All procedures involving animals were in accordance with the institutional animal welfare guidelines of the Chung Shan Medical University. For the nude mice xenograft model, 5-6 weeks old immunodeficient nude mice (BALB/c nu/nu mice) weighing 18-22 $\mathrm{g}$ were used. The mice were housed

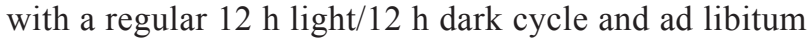
access to standard rodent chow diet (Laboratory Rodent Diet 5001, LabDiet, St. Louis, MO) and were kept in a pathogen-free environment at the Laboratory Animal Unit. SAS-derived sphere-forming OSCC-CSCs $(5 \times$ $10^{4}$ cells $/ 0.2 \mathrm{~mL} /$ mouse) were injected subcutaneously into the right front axilla. Eight days postimplantation, the mice were randomly divided into two groups $(\mathrm{N}=$ 6 for each group) and fed by oral gavage with saline (control) and ISL $(5 \mathrm{mg} / \mathrm{day} / \mathrm{kg}$ ) suspended in saline. The day of cell implantation was designated day 0 . Bioluminescence imaging (BLI) was performed using an IVIS50 animal imaging system (Xenogen Corp., Alameda, CA, USA). The photons emitted from the target site penetrated through the mammalian tissue and could be externally detected and quantified using a sensitive light imaging system. The image acquisition time was $1 \mathrm{~min}$. The displayed images of the tumor sites were drawn around and quantified in photons per second using Living Image software (Xenogen Corp.) The volume was calculated (according to the following formula: ([length $\times$ width $\left.^{2}\right] / 2$ ) and then analyzed using Image-Pro Plus software. After 20 days, the animals were euthanized, and the primary tumors were isolated and weighed [57].

\section{Statistical analysis}

SPSS (version 13.0) was used for statistical analysis. Student's $t$ test or ANOVA analysis were used to determine the statistical significance of the differences between experimental groups; $p$ values less than 0.05 were considered statistically significant.

\section{FUNDING}

This study was supported by grants from Ministry of Science and Technology (MOST 105-2320-B-039003-MY2), Chung Shan Medical University Hospital (CSH-2017-C-005), and China Medical University (CMU105-S-45) in Taiwan.

\section{CONFLICTS OF INTEREST}

The authors have declared no conflicts of interest.

\section{REFERENCES}

1. Warnakulasuriya S. Global epidemiology of oral and oropharyngeal cancer. Oral Oncol. 2009; 45: 309-16.

2. Gibson MK, Forastiere AA. Reassessment of the role of induction chemotherapy for head and neck cancer. Lancet Oncol. 2006; 7: 565-74.

3. Hasegawa T, Shibuya Y, Takeda D, Iwata E, Saito I, Kakei Y, Sakakibara A, Akashi M, Minamikawa T, Komori T. Prognosis of oral squamous cell carcinoma patients with level IV/V metastasis: an observational study. J Craniomaxillofac Surg. 2017; 45: 145-9.

4. Morrison R, Schleicher SM, Sun Y, Niermann KJ, Kim S, Spratt DE, Chung CH, Lu B. Targeting the mechanisms of resistance to chemotherapy and radiotherapy with the cancer stem cell hypothesis. J Oncol. 2011; 2011: 941876.

5. Magee JA, Piskounova E, Morrison SJ. Cancer stem cells: impact, heterogeneity, and uncertainty. Cancer Cell. 2012; 21: 283-96.

6. Medema JP. Cancer stem cells: the challenges ahead. Nat Cell Biol. 2013; 15: 338-44.

7. Vidal SJ, Rodriguez-Bravo V, Galsky M, Cordon-Cardo C, Domingo-Domenech J. Targeting cancer stem cells to suppress acquired chemotherapy resistance. Oncogene. 2014; 33: 4451-63.

8. Sotiropoulou PA, Christodoulou MS, Silvani A, HeroldMende C, Passarella D. Chemical approaches to targeting drug resistance in cancer stem cells. Drug Discov Today. 2014; 19: 1547-62.

9. Peitzsch C, Tyutyunnykova A, Pantel K, Dubrovska A. Cancer stem cells: the root of tumor recurrence and metastases. Sem Cancer Biol. 2017; 44: 10-24.

10. Chen G, Zhu L, Liu Y, Zhou Q, Chen H, Yang J. Isoliquiritigenin, a flavonoid from licorice, plays a dual role in regulating gastrointestinal motility in vitro and in vivo. Phytother Res. 2009; 23: 498-506.

11. Sato Y, He JX, Nagai H, Tani T, Akao T. Isoliquiritigenin, one of the antispasmodic principles of Glycyrrhiza ularensis 
roots, acts in the lower part of intestine. Biol Pharm Bull. 2007; 30: 145-9.

12. Shi Y, Wu D, Sun Z, Yang J, Chai H, Tang L, Guo Y. Analgesic and uterine relaxant effects of isoliquiritigenin, a flavone from Glycyrrhiza glabra. Phytother Res. 2012; 26: $1410-7$.

13. Yu SM, Kuo SC. Vasorelaxant effect of isoliquiritigenin, a novel soluble guanylate cyclase activator, in rat aorta. Br J Pharmacol. 1995; 114: 1587-94.

14. Liu B, Yang J, Wen Q, Li Y. Isoliquiritigenin, a flavonoid from licorice, relaxes guinea-pig tracheal smooth muscle in vitro and in vivo: role of cGMP/PKG pathway. Eur J Pharmacol. 2008; 587: 257-66.

15. Kim JY, Park SJ, Yun KJ, Cho YW, Park HJ, Lee KT. Isoliquiritigenin isolated from the roots of Glycyrrhiza uralensis inhibits LPS-induced iNOS and COX-2 expression via the attenuation of NF- $\kappa \mathrm{B}$ in RAW 264.7 macrophages. Eur J Pharmacol. 2008; 584: 175-84.

16. Wang Z, Wang N, Han S, Wang D, Mo S, Yu L, Huang H, Tsui K, Shen J, Chen J. Dietary compound isoliquiritigenin inhibits breast cancer neoangiogenesis via VEGF/VEGFR-2 signaling pathway. PLoS One. 2013; 8: e68566.

17. Takahashi $T$, Baba M, Nishino $H$, Okuyama $T$. Cyclooxygenase-2 plays a suppressive role for induction of apoptosis in isoliquiritigenin-treated mouse colon cancer cells. Cancer Lett. 2006; 231: 319-25.

18. Si L, Yang X, Yan X, Wang Y, Zheng Q. Isoliquiritigenin induces apoptosis of human bladder cancer T24 cells via a cyclin-dependent kinase-independent mechanism. Oncol Lett. 2017; 14: 241-9.

19. Kim DH, Park JE, Chae IG, Park G, Lee S, Chun KS. Isoliquiritigenin inhibits the proliferation of human renal carcinoma Caki cells through the ROS-mediated regulation of the Jak2/STAT3 pathway. Oncol Rep. 2017; 38: 575-83.

20. Wu CH, Chen HY, Wang CW, Shieh TM, Huang TC, Lin LC, Wang KL, Hsia SM. Isoliquiritigenin induces apoptosis and autophagy and inhibits endometrial cancer growth in mice. Oncotarget. 2016; 7: 73432-47. https://doi. org/10.18632/oncotarget.12369.

21. Jung SK, Lee MH, Lim DY, Kim JE, Singh P, Lee SY, Jeong CH, Lim TG, Chen H, Chi YI, Kundu JK, Lee NH, Lee $\mathrm{CC}$, et al. Isoliquiritigenin induces apoptosis and inhibits xenograft tumor growth of human lung cancer cells by targeting both wild type and L858R/T790M mutant EGFR. J Biol Chem. 2014; 289: 35839-48.

22. Hou C, Li W, Li Z, Gao J, Chen Z, Zhao X, Yang Y, Zhang $\mathrm{X}$, Song Y. Synthetic isoliquiritigenin inhibits human tongue squamous carcinoma cells through its antioxidant mechanism. Oxid Med Cell Longev. 2017; 2017: 1379430.

23. Hsia SM, Yu CC, Shih YH, Yuanchien Chen M, Wang TH, Huang YT, Shieh TM. Isoliquiritigenin as a cause of DNA damage and inhibitor of ataxia-telangiectasia mutated expression leading to $\mathrm{G} 2 / \mathrm{M}$ phase arrest and apoptosis in oral squamous cell carcinoma. Head Neck. 2016; 38: E360-71.
24. Wang N, Wang Z, Wang Y, Xie X, Shen J, Peng C, You J, Peng F, Tang H, Guan X, Chen J. Dietary compound isoliquiritigenin prevents mammary carcinogenesis by inhibiting breast cancer stem cells through WIF1 demethylation. Oncotarget. 2015; 6: 9854-76. https://doi. org/10.18632/oncotarget.3396.

25. Wang N, Wang Z, Peng C, You J, Shen J, Han S, Chen J. Dietary compound isoliquiritigenin targets GRP78 to chemosensitize breast cancer stem cells via $\beta$-catenin/ ABCG2 signaling. Carcinogenesis. 2014; 35: 2544-54.

26. Yin Y, Chen C, Chen J, Zhan R, Zhang Q, Xu X, Li D, Li M. Cell surface GRP78 facilitates hepatoma cells proliferation and migration by activating IGF-IR. Cell Signal. 2017; 35 : 154-62.

27. Lizardo MM, Morrow JJ, Miller TE, Hong ES, Ren L, Mendoza A, Halsey CH, Scacheri PC, Helman LJ, Khanna C. Upregulation of glucose-regulated protein 78 in metastatic cancer cells is necessary for lung metastasis progression. Neoplasia. 2016; 18: 699-710.

28. Gu YJ, Li HD, Zhao L, Zhao S, He WB, Rui L, Su C, Zheng HC, Su RJ. GRP78 confers the resistance to 5-FU by activating the c-Src/LSF/TS axis in hepatocellular carcinoma. Oncotarget. 2015; 6: 33658-74. https://doi. org/10.18632/oncotarget.5603.

29. Berger CL, Dong Z, Hanlon D, Bisaccia E, Edelson RL. A lymphocyte cell surface heat shock protein homologous to the endoplasmic reticulum chaperone, immunoglobulin heavy chain binding protein BIP. Int J Cancer. 1997; 71: 1077-85.

30. Misra UK, Deedwania R, Pizzo SV. Activation and crosstalk between Akt, NF-kappaB, and unfolded protein response signaling in 1-LN prostate cancer cells consequent to ligation of cell surface-associated GRP78. J Biol Chem. 2006; 281: 13694-707.

31. Lee SH, Koo BS, Kim JM, Huang S, Rho YS, Bae WJ, Kang HJ, Kim YS, Moon JH, Lim YC. Wnt//-catenin signalling maintains self-renewal and tumourigenicity of head and neck squamous cell carcinoma stem-like cells by activating Oct4. J Pathol. 2014; 234: 99-107.

32. O'Brien CA, Kreso A, Jamieson CH. Cancer stem cells and self-renewal. Clin Cancer Res. 2010; 16: 3113-20.

33. Chen SF, Chang YC, Nieh S, Liu CL, Yang CY, Lin YS. Nonadhesive culture system as a model of rapid sphere formation with cancer stem cell properties. PLoS One. 2012; 7: e31864.

34. Lo JF, Yu CC, Chiou SH, Huang CY, Jan CI, Lin SC, Liu CJ, Hu WY, Yu YH. The epithelial-mesenchymal transition mediator S100A4 maintains cancer-initiating cells in head and neck cancers. Cancer Res. 2011; 71: 1912-23.

35. Chen YC, Chen YW, Hsu HS, Tseng LM, Huang PI, Lu KH, Chen DT, Tai LK, Yung MC, Chang SC, Ku HH, Chiou $\mathrm{SH}$, Lo WL. Aldehyde dehydrogenase 1 is a putative marker for cancer stem cells in head and neck squamous cancer. Biochem Biophys Res Commun. 2009; 385: 307-13. 
36. Yu CC, Lo WL, Chen YW, Huang PI, Hsu HS, Tseng LM, Hung SC, Kao SY, Chang CJ, Chiou SH. Bmi-1 regulates Snail expression and promotes metastasis ability in head and neck squamous cancer-derived ALDH1 positive cells. J Oncol. 2011; 2011: 609259.

37. Morgan CA, Parajuli B, Buchman CD, Dria K, Hurley TD. $\mathrm{N}, \mathrm{N}$-diethylaminobenzaldehyde (DEAB) as a substrate and mechanism-based inhibitor for human ALDH isoenzymes. Chem Biol Interact. 2015; 234: 18-28.

38. Prince ME, Sivanandan R, Kaczorowski A, Wolf GT, Kaplan MJ, Dalerba P, Weissman IL, Clarke MF, Ailles LE. Identification of a subpopulation of cells with cancer stem cell properties in head and neck squamous cell carcinoma. Proc Natl Acad Sci U S A. 2007; 104: 973-8.

39. Brabletz T, Jung A, Spaderna S, Hlubek F, Kirchner T. Opinion: migrating cancer stem cells - an integrated concept of malignant tumour progression. Nat Rev Cancer. 2005; 5: 744-9.

40. Holohan C, Van Schaeybroeck S, Longley DB, Johnston PG. Cancer drug resistance: an evolving paradigm. Nat Rev Cancer. 2013; 13: 714-26.

41. Dean M, Fojo T, Bates S. Tumour stem cells and drug resistance. Nat Rev Cancer. 2005; 5: 275-84.

42. An Y, Ongkeko WM. ABCG2: the key to chemoresistance in cancer stem cells? Exp Opin Drug Metab Toxicol. 2009; 5: $1529-42$.

43. Balbuena J, Pachon G, Lopez-Torrents G, Aran JM, Castresana JS, Petriz J. ABCG2 is required to control the sonic hedgehog pathway in side population cells with stemlike properties. Cytometry A. 2011; 79: 672-83.

44. Wu MJ, Jan CI, Tsay YG, Yu YH, Huang CY, Lin SC, Liu CJ, Chen YS, Lo JF, Yu CC. Elimination of head and neck cancer initiating cells through targeting glucose regulated protein78 signaling. Mol Cancer. 2010; 9: 283.

45. Baccelli I, Trumpp A. The evolving concept of cancer and metastasis stem cells. J Cell Biol. 2012; 198: 281-93.

46. Abdullah LN, Chow EK. Mechanisms of chemoresistance in cancer stem cells. Clin Transl Med. 2013; 2: 3.

47. Dong D, Ni M, Li J, Xiong S, Ye W, Virrey JJ, Mao C, Ye R, Wang M, Pen L, Dubeau L, Groshen S, Hofman FM, et al. Critical role of the stress chaperone GRP78/BiP in tumor proliferation, survival, and tumor angiogenesis in transgeneinduced mammary tumor development. Cancer Res. 2008; 68: 498-505.

48. Dong D, Stapleton C, Luo B, Xiong S, Ye W, Zhang Y, Jhaveri N, Zhu G, Ye R, Liu Z, Bruhn KW, Craft N, Groshen S, et al. A critical role for GRP78/BiP in the tumor microenvironment for neovascularization during tumor growth and metastasis. Cancer Res. 2011; 71: 2848-57.

49. Kuo LJ, Hung CS, Chen WY, Chang YJ, Wei PL. Glucoseregulated protein 78 silencing down-regulates vascular endothelial growth factor/vascular endothelial growth factor receptor 2 pathway to suppress human colon cancer tumor growth. J Surg Res. 2013; 185: 264-72.

50. Yeung BH, Kwan BW, He QY, Lee AS, Liu J, Wong AS. Glucose-regulated protein 78 as a novel effector of BRCA1 for inhibiting stress-induced apoptosis. Oncogene. 2008; 27: 6782-9.

51. Lee HK, Xiang C, Cazacu S, Finniss S, Kazimirsky G, Lemke N, Lehman NL, Rempel SA, Mikkelsen T, Brodie C. GRP78 is overexpressed in glioblastomas and regulates glioma cell growth and apoptosis. Neuro Oncol. 2008; 10: 236-43.

52. Pyrko P, Schönthal AH, Hofman FM, Chen TC, Lee AS. The unfolded protein response regulator GRP78/BiP as a novel target for increasing chemosensitivity in malignant gliomas. Cancer Res. 2007; 67: 9809-16.

53. Li Y, Zhao H, Wang Y, Zheng H, Yu W, Chai H, Zhang J, Falck JR, Guo AM, Yue J, Peng R, Yang J. Isoliquiritigenin induces growth inhibition and apoptosis through downregulating arachidonic acid metabolic network and the deactivation of PI3K/Akt in human breast cancer. Toxicol Appl Pharmacol. 2013; 272: 37-48.

54. Kelber JA, Panopoulos AD, Shani G, Booker EC, Belmonte JC, Vale WW, Gray PC. Blockade of Cripto binding to cell surface GRP78 inhibits oncogenic Cripto signaling via MAPK/PI3K and Smad2/3 pathways. Oncogene. 2009; 28 : 2324-36.

55. Fu Y, Wey S, Wang M, Ye R, Liao CP, Roy-Burman P, Lee AS. Pten null prostate tumorigenesis and AKT activation are blocked by targeted knockout of ER chaperone GRP78/ BiP in prostate epithelium. Proc Natl Acad Sci U S A. 2008; 105: 19444-9.

56. Yu CC, Chen PN, Peng CY, Yu CH, Chou MY. Suppression of miR-204 enables oral squamous cell carcinomas to promote cancer stemness, EMT traits, and lymph node metastasis. Oncotarget. 2016; 7: 20180-92. https://doi. org/10.18632/oncotarget.7745.

57. Hu FW, Tsai LL, Yu CH, Chen PN, Chou MY, Yu CC. Impairment of tumor-initiating stem-like property and reversal of epithelial-mesenchymal transdifferentiation in head and neck cancer by resveratrol treatment. Mol Nutr Food Res. 2012; 56: 1247-58.

58. $\mathrm{Yu} \mathrm{CH}, \mathrm{Yu} \mathrm{CC}$. Photodynamic therapy with 5-aminolevulinic acid (ALA) impairs tumor initiating and chemo-resistance property in head and neck cancer-derived cancer stem cells. PLoS One. 2014; 9: e87129.

59. Yang PY, Hsieh PL, Wang TH, Yu CC, Lu MY, Liao YW, Lee TH, Peng CY. Andrographolide impedes cancer stemness and enhances radio-sensitivity in oral carcinomas via miR-218 activation. Oncotarget. 2017; 8: 4196-207. https://doi.org/10.18632/oncotarget.13755.

60. Yu CC, Hu FW, Yu CH, Chou MY. Targeting CD133 in the enhancement of chemosensitivity in oral squamous cell 
carcinoma-derived side population cancer stem cells. Head Neck. 2016; 38: E231-8.

61. Huang CE, Hu FW, Yu CH, Tsai LL, Lee TH, Chou MY, Yu CC. Concurrent expression of Oct4 and Nanog maintains mesenchymal stem-like property of human dental pulp cells. Int J Mol Sci. 2014; 15: 18623-39.
62. Chou MY, Hu FW, Yu CH, Yu CC. Sox2 expression involvement in the oncogenicity and radiochemoresistance of oral cancer stem cells. Oral Oncol. 2015; 51: 31-9. 